\title{
Workspace Analysis for a Kinematically Coupled Torso of a Torque Controlled Humanoid Robot
}

\author{
Alexander Dietrich ${ }^{1}$, Melanie Kimmel ${ }^{2}$, Thomas Wimböck ${ }^{1}$, Sandra Hirche ${ }^{2}$, and Alin Albu-Schäffer ${ }^{1}$ \\ ${ }^{1}$ DLR - German Aerospace Center, Germany \\ ${ }^{2}$ TUM - Technische Universität München, Germany
}

\begin{abstract}
The workspace and performance of a humanoid robot is decisively influenced by the design of its torso. The joints or spinal discs are usually the weak points due to the high stress they are exposed to, e.g. when lifting heavy objects. One way to circumvent the necessity of large motors is to use parallel mechanisms to optimize the distribution of loads. Here, we analyze the workspace of the humanoid robot Rollin' Justin of the German Aerospace Center (DLR) w. r.t. the constraints imposed by kinematic coupling of torso joints via tendons. The results of the analysis can be used for planning and reactive control to efficiently exploit the torso performance capabilities of the robotic system. As an application, we design a potential field based controller to avoid violating these constraints and implement it on the real robot.
\end{abstract}

\section{INTRODUCTION}

In humanoid robot design, the focus is often on hands for grasping, arms for manipulation, and legs for walking. Only little attention is paid to torso design, although crucial capabilities of the system are determined by the trunk. In [1] it is shown that, while walking, energy consumption of legged humanoids can be reduced by $26.5 \%$ when exploiting the torso dynamics in contrast to a rigid torso structure. Another example for the major influence of the trunk is the overall workspace of the robot. The torso of TwendyOne [2] with four degrees of freedom (DOF) allows to grasp objects from the floor. The same applies to Justin [3] or Kotaro [4], for example. Many tasks in service robotics require both a large workspace of the end-effectors and kinematic redundancy, e.g. because of several simultaneous objectives or constrained motions such as opening a door or a drawer. Instead of mainly moving the base of a mobile manipulator [5], a versatile torso can support a lot to increase the workspace. And there are even tasks in which an articulated torso is inevitable. Consider a remote object that can only be grasped by reaching across a table [6].

However, articulated torsos also raise several problems. Beside increased complexity in terms of the design (electronics, power supply, control), the joints are usually exposed to high torques. Consider the long lever arm when lifting an object far away from the hip, for example. That requires very large motors. One solution is to apply parallel structures in order to optimize the distribution of loads. The robots Kotaro [4] and Kojiro [7] use artificial muscles to actuate a flexible-spine structure. At DLR, the wheeled humanoid

Contact: German Aerospace Center (DLR), Institute of Robotics and Mechatronics, Wessling D-82230. Alexander.Dietrich@dlr.de

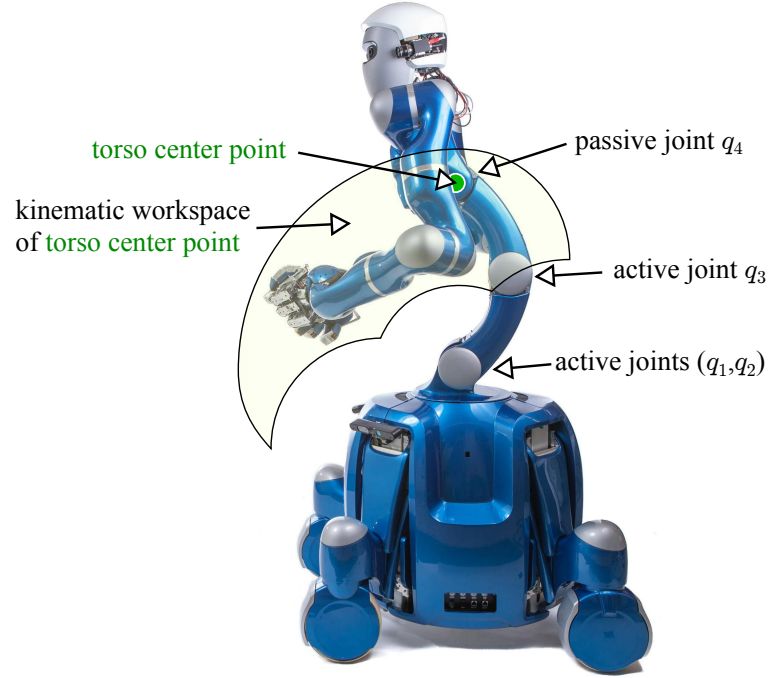

Fig. 1. The kinematic workspace of the torso center point (shaded area) is a result of the restricted motion of the passive joint due to the tendon coupling in the torso.

Justin [3] (Fig. 1) has been designed with three actuated torso joints and a kinematically coupled fourth one. This coupling via tendons allows to counteract the torques about the horizontal axes, see Fig. 2 [8]. Hence, small motors can be used in the upper body, and that complies well with the lightweight design principles of Justin. The holonomic constraint imposed by the tendon routing restricts, however, the kinematic workspace. That is illustrated by the possible locations of the torso center point in Fig. 1. The joint torques are also influenced by that coupling and have to be accounted for during operation.

In this paper, we will analyze the kinematic and dynamic ${ }^{1}$ torso workspace of Justin exemplarily for such a kinematic coupling. Closing the gap between robot performance and its human archetype requires dealing with this subject, in particular when considering the impressive workspace of a human being. We represent the imposed constraints on the torso in the intuitive Cartesian space of the torso center point (Fig. 1). The analysis is useful for motion planning and control. Another contribution of this paper is the ex-

\footnotetext{
${ }^{1}$ The dynamic constraints (torque limit constraints) are determined by instantaneous forces arising from the dynamic equations (inertial, Coriolis/centrifugal, gravity, and external forces).
} 
perimental evaluation of a controller which repels the torso from its kinematic and dynamic workspace boundaries. The experiments are conducted on Justin.

The paper is organized as follows: After a brief introduction to the specific mechanical design of the torso of Justin in Sec. II-A, the kinematic and the dynamic constraints on its workspace are derived in Sec. II-B and Sec. II-C, respectively. Afterwards, Sec. II-D shows applications for the performed analysis. One of these applications, a potential field based controller (Sec. II-E), is experimentally validated on the real robot in Sec. III.

\section{THE TORSO WORKSPACE OF JUSTIN}

In this section, the structure of the torso of Justin is introduced and the workspace boundaries are derived from the mathematical equations which arise from the kinematic constraints and the actuator limitations.

\section{A. Model of the Torso}

The torso of Justin contains one passive and three actuated joints, see Fig. 1. Tendons are used to kinematically couple the torso joints $q_{2}, q_{3}$, and $q_{4}$. The vertical axis with joint value $q_{1}$ can be treated separately because it is not kinematically coupled. Fig. 2 depicts the tendon routing. Motors are placed at $q_{2}$ and $q_{3}$. The upper joint $q_{4}$ is passive and ensures that the chest is always kept in an upright configuration. Assuming that the tendons are inelastic, the kinematic, linear, holonomic constraint

$$
\boldsymbol{A} \dot{\boldsymbol{q}}=\mathbf{0}
$$

with $\boldsymbol{A}=\left[\begin{array}{llll}0 & 1 & 1 & 1\end{array}\right]$ and $\dot{\boldsymbol{q}}=\left[\begin{array}{llll}\dot{q}_{1} & \dot{q}_{2} & \dot{q}_{3} & \dot{q}_{4}\end{array}\right]^{T}$ holds true. The main advantage of this concept is that load torques, which occur at the chest, can be redirected to the robot base without motor work. Of course, this tendon coupling introduces elasticities so that the kinematic constraint of an upright torso is an assumption that would only hold for infinite tendon stiffness. However, experimental evaluations revealed that the joint angle deflections are very small and negligible in this context. Any force or torque applied at the end-effector (or anywhere else on the robot) can easily be projected back into the torso center point. There, only forces in the $x-z$-plane have to be compensated by active control. So considering only the $x$-z-plane according to Fig. 2 simplifies the calculations but does not disregard any relevant physical effects. More detailed information on the mechanics of the torso can be found in [8], [9].

\section{B. Kinematic Constraints}

According to Fig. 2, the Cartesian coordinates of the torso center point in the $x$ - $z$-plane are

$$
\boldsymbol{p}(\boldsymbol{q})=\left(\begin{array}{l}
p_{\mathrm{x}}(\boldsymbol{q}) \\
p_{\mathrm{z}}(\boldsymbol{q})
\end{array}\right)=\left(\begin{array}{l}
l_{2} \sin \left(q_{2}\right)+l_{3} \sin \left(q_{2}+q_{3}\right) \\
l_{2} \cos \left(q_{2}\right)+l_{3} \cos \left(q_{2}+q_{3}\right)
\end{array}\right)
$$

and the Jacobian matrix can be derived as

$$
\begin{aligned}
\boldsymbol{J}(\boldsymbol{q}) & =\left(\begin{array}{ll}
\frac{\partial \boldsymbol{p}(\boldsymbol{q})}{\partial q_{2}} & \frac{\partial \boldsymbol{p}(\boldsymbol{q})}{\partial q_{3}}
\end{array}\right) \\
& =\left(\begin{array}{cc}
l_{2} \cos \left(q_{2}\right)+l_{3} \cos \left(q_{2}+q_{3}\right) & l_{3} \cos \left(q_{2}+q_{3}\right) \\
-l_{2} \sin \left(q_{2}\right)-l_{3} \sin \left(q_{2}+q_{3}\right) & -l_{3} \sin \left(q_{2}+q_{3}\right)
\end{array}\right)
\end{aligned}
$$

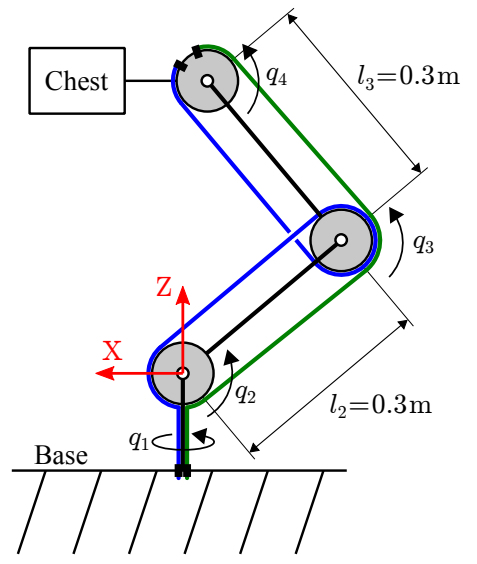

Fig. 2. Sketch of the torso illustrating the tendon kinematics [9]. In the zero configuration, the two torso links are aligned with the vertical axis.

TABLE I

JOINT RANGES FOR THE ACTUATED TORSO JOINTS OF JUSTIN [8].

\begin{tabular}{|c|c|c|}
\hline Joint & Minimum value & Maximum value \\
\hline \hline$q_{1}$ & $-140^{\circ}$ & $200^{\circ}$ \\
\hline$q_{2}$ & $-90^{\circ}$ & $90^{\circ}$ \\
\hline$q_{3}$ & $\max \left(0^{\circ},-q_{2}\right)$ & $\min \left(135^{\circ}, 135^{\circ}-q_{2}\right)$ \\
\hline
\end{tabular}

to relate the Cartesian and the joint space in a differential way. In Table I, the mechanical end stops of the joints are listed. These joint ranges along with (2) determine the kinematic workspace of the torso center point depicted in Fig. 3, [8]. The analytical expressions of the workspace boundaries are provided in Table II.

\section{Dynamic Constraints}

Depending on the configuration of the torso, a load

$$
\boldsymbol{F}=\left(\begin{array}{c}
F_{x} \\
F_{z}
\end{array}\right)=F_{\text {load }}\left(\begin{array}{c}
\cos (\alpha) \\
-\sin (\alpha)
\end{array}\right)
$$

which results at the torso center point requires motor torques to counteract:

$$
\begin{aligned}
\boldsymbol{\tau} & =-\boldsymbol{J}(\boldsymbol{q})^{T} \boldsymbol{F} \\
& =-F_{\text {load }}\left(\begin{array}{c}
l_{3} \cos \left(q_{2}+q_{3}-\alpha\right)+l_{2} \cos \left(q_{2}-\alpha\right) \\
l_{3} \cos \left(q_{2}+q_{3}-\alpha\right)
\end{array}\right) .
\end{aligned}
$$

TABLE II

CENTERS AND RADII OF THE ARCS DEFINING THE KINEMATIC WORKSPACE BOUNDARIES OF THE TORSO OF JUSTIN.

\begin{tabular}{|c|c|c|c|}
\hline Arc & Constraint & Center & Radius \\
\hline \hline 1 & $q_{2}=-90^{\circ}$ & $\left(-l_{2}, 0\right)$ & $l_{3}$ \\
\hline 2 & $q_{3}=135^{\circ}$ & $(0,0)$ & $\sqrt{l_{2}^{2}+l_{3}^{2}-\sqrt{2} l_{2} l_{3}}$ \\
\hline 3 & $q_{3}=135^{\circ}-q_{2}$ & $\left(l_{3} / \sqrt{2},-l_{3} / \sqrt{2}\right)$ & $l_{2}$ \\
\hline 4 & $q_{2}=90^{\circ}$ & $\left(l_{2}, 0\right)$ & $l_{3}$ \\
\hline 5 & $q_{3}=0^{\circ}$ & $(0,0)$ & $l_{2}+l_{3}$ \\
\hline 6 & $q_{3}=-q_{2}$ & $\left(0, l_{3}\right)$ & $l_{2}$ \\
\hline
\end{tabular}




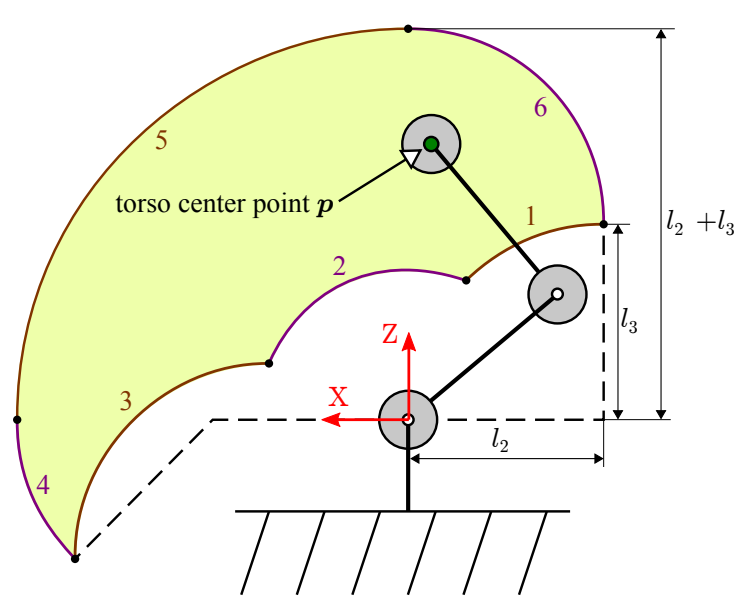

Fig. 3. The workspace of the torso center point (yellow/shaded area) is bounded by the kinematics of the tendons. The numbers 1-6 correspond to the arcs in Table II.

The angle $\alpha$ is defined about the $y$-axis. The range of feasible motor torques

$$
\begin{aligned}
& \tau_{2, \text { min }} \leq \tau_{2} \leq \tau_{2, \text { max }} \\
& \tau_{3, \text { min }} \leq \tau_{3} \leq \tau_{3, \text { max }}
\end{aligned}
$$

additionally constrains the workspace of the torso if a load force is applied via (5). These restrictions are called dynamic constraints because the actual force $\boldsymbol{F}$ is directly linked to the dynamic equations of the torso. In other words, the constraints incorporate gravitational effects (weight of the upper body, tools), inertial effects, external forces (contact of the upper body with the environment), and Coriolis/centrifugal effects. Moreover, these constraints are not invariant like the kinematic ones in Fig. 3, but they dynamically change depending on the actual value of $\boldsymbol{F}$. That characteristic is shown in the attached video.

The boundaries concerning $\tau_{2}$ can be derived from (5) (first line) by inserting the forward kinematics (2). That leads to

$$
\frac{\tau_{2}}{F_{\text {load }}}=-x \sin (\alpha)-z \cos (\alpha)
$$

which represents straight lines in the $x$ - $z$-plane. Inserting the boundaries $\tau_{2 \text {,min }}$ and $\tau_{2 \text {,max }}$ from (6) delivers the dynamic workspace constraints on the Cartesian coordinates of the torso center point. Their geometric interpretation is very intuitive: The ratios $\tau_{2, \min } / F_{\text {load }}$ and $\tau_{2 \text {,max }} / F_{\text {load }}$ determine the distance between the boundaries while $\alpha$ describes the decline of the belt for feasible torques in torso joint two. Examples for this belt are shown in Fig. 4 for different parameterizations of $F_{\text {load }}$ and $\alpha$.

The boundaries for $\tau_{3}$ can be derived by solving (5) (second line) for $q_{2}+q_{3}$, which delivers

$$
q_{2}+q_{3}=\alpha \pm \arccos \left(\frac{-\tau_{3}}{F_{\text {load }} l_{3}}\right) .
$$

Once again, one can apply the limits on the motor torque, i.e. $\tau_{3, \min }$ and $\tau_{3, \max }$ from (7). If $\tau_{3}, \alpha$, and $F_{\text {load }}$ are constant, then $q_{2}+q_{3}$ is evidently constant too. Eq. (9) states that there only exists a configuration if

$$
-1 \leq-\frac{\tau_{3, \min }}{F_{\text {load }} l_{3}} \leq 1, \quad \text { or } \quad-1 \leq-\frac{\tau_{3, \max }}{F_{\text {load }} l_{3}} \leq 1 .
$$

If these inequalities are not fulfilled, no constraints on the workspace are imposed by the actuator limits at the third torso joint. That effect can be interpreted as the minimum/maximum torque being sufficient to counteract the given load force in any part of the kinematic workspace. Analogous to (8), one can express the workspace boundaries in the $x$ - $z$-representation by applying the forward kinematics (2) again.

$$
\left(x-l_{3} \sin \left(q_{2}+q_{3}\right)\right)^{2}+\left(z-l_{3} \cos \left(q_{2}+q_{3}\right)\right)^{2}=l_{2}^{2} .
$$

This constraint is geometrically described by circles with radius $l_{2}$ and corresponding centers at

$$
\begin{aligned}
& x=l_{3} \sin \left(\alpha \pm \arccos \left(\frac{-\tau_{3, \text { min }}}{F_{\text {load }} l_{3}}\right)\right), \\
& z=l_{3} \cos \left(\alpha \pm \arccos \left(\frac{-\tau_{3, \text { min }}}{F_{\text {load }} l_{3}}\right)\right),
\end{aligned}
$$

and

$$
\begin{aligned}
& x=l_{3} \sin \left(\alpha \pm \arccos \left(\frac{-\tau_{3, \max }}{F_{\text {load }} l_{3}}\right)\right), \\
& z=l_{3} \cos \left(\alpha \pm \arccos \left(\frac{-\tau_{3, \text { max }}}{F_{\text {load }} l_{3}}\right)\right) .
\end{aligned}
$$

Due to the cases (9) and the torque limits $\tau_{3, \min }(12)-(13)$ and $\tau_{3, \max }(14)-(15)$, four boundaries of this type exist. More details on the center locations and workspace specifications are provided in Table III.

The four circle centers span a rectangle. According to (12)-(15), their locations depend on the load as well as on the torque limits. In case of $\tau_{2, \text { min }}=\tau_{3, \text { min }}=-\tau_{2, \max }=$ $-\tau_{3, \max }$, the centers lie on the straight $\tau_{2}$-boundaries. That can easily be proven by inserting (12)-(15) into (8). The circular boundaries are illustrated in Fig. 4, and the overall workspace results from intersecting the kinematic and the dynamic workspace of the torso. The four scenarios represent different loads with different effective directions for

$$
\begin{aligned}
& -230 \mathrm{Nm} \leq \tau_{2} \leq 230 \mathrm{Nm}, \\
& -230 \mathrm{Nm} \leq \tau_{3} \leq 230 \mathrm{Nm} .
\end{aligned}
$$

The $\tau_{2}$-boundaries are parallel to the direction of $\boldsymbol{F}$, cf. (8). The circular boundaries are actually defined by semicircles instead of full circles. This is due to the fact that $q_{3} \geq 0$ always holds according to Table I. Only one half of each circle ensures a constant angle sum $q_{2}+q_{3}$ (cf. (9) and (11)) that complies both with the holonomic constraint (1) and $q_{3} \geq 0$. Invalid workspace areas exist between two circular constraint boundaries based on the same minimum (or maximum) torque of the joint. There, the joint torque would exceed the maximum torque or go below the minimum torque, respectively. If $F_{\text {load }}$ gets smaller, the semicircles approach each other (Fig. 4c $\rightarrow$ Fig. 4a), hence the workspace 
TABLE III

DYNAMIC WORKSPACE CONSTRAINTS DUE TO TORQUE LIMITS IN THE THIRD TORSO JOINT.

\begin{tabular}{|c|c|c|c|}
\hline$\tau_{3}$ & $x$-Value of Center & $z$-Value of Center & Workspace \\
\hline \hline$\tau_{3, \min }$ & $l_{3} \sin \left(\alpha+\arccos \left(\frac{-\tau_{3, \min }}{F_{\text {load }} l_{3}}\right)\right)$ & $l_{3} \cos \left(\alpha+\arccos \left(\frac{-\tau_{3, \text { min }}}{F_{\text {load }} l_{3}}\right)\right)$ & inside \\
\hline$\tau_{3, \min }$ & $l_{3} \sin \left(\alpha-\arccos \left(\frac{-\tau_{3, \text { min }}}{F_{\text {load }} l_{3}}\right)\right)$ & $l_{3} \cos \left(\alpha-\arccos \left(\frac{-\tau_{3, \text { min }}}{F_{\text {load }} l_{3}}\right)\right)$ & outside \\
\hline$\tau_{3, \max }$ & $l_{3} \sin \left(\alpha+\arccos \left(\frac{-\tau_{3, \max }}{F_{\text {load }} l_{3}}\right)\right)$ & $l_{3} \cos \left(\alpha+\arccos \left(\frac{-\tau_{3, \text { max }}}{F_{\text {load }} l_{3}}\right)\right)$ & outside \\
\hline$\tau_{3, \max }$ & $l_{3} \sin \left(\alpha-\arccos \left(\frac{-\tau_{3, \max }}{F_{\text {load }} l_{3}}\right)\right)$ & $l_{3} \cos \left(\alpha-\arccos \left(\frac{-\tau_{3, \text { max }}}{F_{\text {load }} l_{3}}\right)\right)$ & inside \\
\hline
\end{tabular}

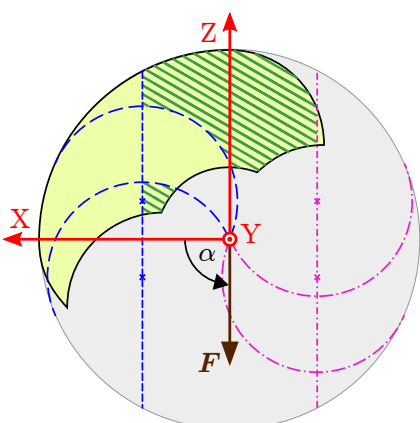

(a) $F_{\text {load }}=800 \mathrm{~N}, \alpha=90^{\circ}$

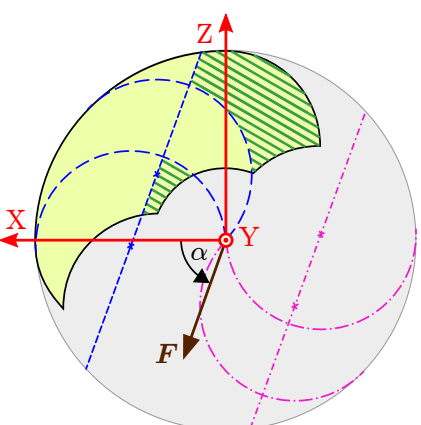

(b) $F_{\text {load }}=800 \mathrm{~N}, \alpha=70^{\circ}$

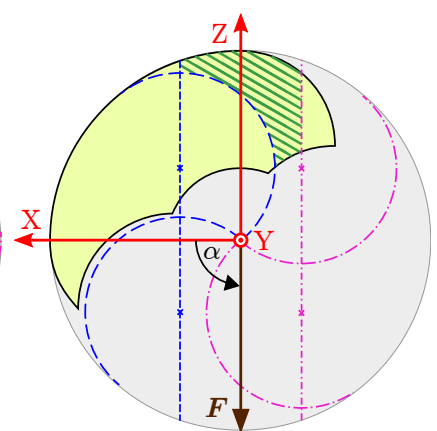

(c) $F_{\text {load }}=1200 \mathrm{~N}, \alpha=90^{\circ}$

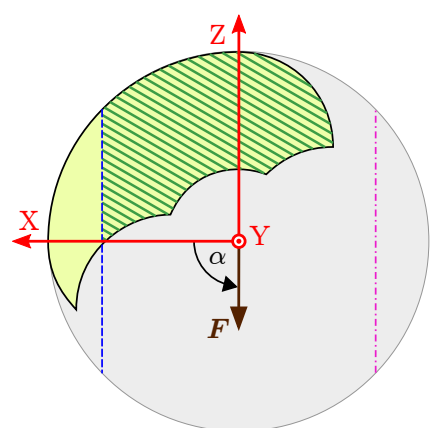

(d) $F_{\text {load }}=550 \mathrm{~N}, \alpha=90^{\circ}$

torso workspace

$------\tau_{2, \min }$ $---\tau_{3, \min } \quad-\cdots \tau_{2, \max } \quad-\cdots-\cdots \tau_{3, \max }$

Fig. 4. Torso workspace of Justin (green/dashed) considering the kinematic and dynamic constraints and a load $\boldsymbol{F}$. The torque limits are set to $\tau_{2, \min }=\tau_{3, \min }=-230 \mathrm{Nm}$ and $\boldsymbol{\tau}_{2, \max }=\tau_{3, \max }=230 \mathrm{Nm}$.

grows. At the point of overlapping, the constraints vanish, see Fig. 4d. The load specification in Fig. 4d with $F_{\text {load }}=550 \mathrm{~N}$ and $\alpha=90^{\circ}$ represents a typical (static) lifting task of an object of about $10 \mathrm{~kg}$. Thereby, the object can easily be picked up from the floor. Even an increase of the object weight to $20 \mathrm{~kg}$ still allows to pick it up from the floor. The attached video illustrates the feasible workspace for a varying load.

\section{Applications of the Analysis}

The knowledge about the kinematic and dynamic constraints on the torso can be used in different applications, in particular for planning and control: The feasibility of torso configurations and the distance to the limits/boundaries can be used as constraints in motion planning algorithms in order to obtain proper and feasible trajectories. One way to deal with the force dependency of the torque constraints (Sec. II-C) is to consider a complete dynamic model of the robot during planning or to set sufficiently large safety margins in case of neglected inertial or other dynamic effects. Another scenario is active control to comply with the constraints. Potential field based approaches [10] are predestined for such an application. Several other objectives have already been accounted for by these techniques on Justin, e. g. selfcollision avoidance [11], Cartesian impedance of the endeffectors [12], singularity avoidance and so forth [13], [14]. Such a controller is explained in the following section.

\section{E. Controller Design}

In a torque controlled mode, repulsive potential fields $V_{\text {rep,total }}(\boldsymbol{q})$ can be used to avoid the kinematic and dynamic boundaries of the torso, e.g. by the control law

$$
\boldsymbol{\tau}=-\left(\frac{\partial V_{\text {rep,total }}(\boldsymbol{q})}{\partial \boldsymbol{q}}\right)^{T}-\boldsymbol{D}(\boldsymbol{q}) \dot{\boldsymbol{q}}+\boldsymbol{g}(\boldsymbol{q}) .
$$

The term $\boldsymbol{g}(\boldsymbol{q})$ compensates for gravitational effects. The positive definite damping matrix

$$
\boldsymbol{D}(\boldsymbol{q})=\mathcal{D}\left(\boldsymbol{M}(\boldsymbol{q}), \frac{\partial^{2} V_{\text {rep,total }}(\boldsymbol{q})}{\partial \boldsymbol{q}^{2}}, \xi\right)
$$

is configuration dependent and allows to realize a particular damping ratio $\xi$ in the direction of the repulsion. The determination of (17) is straightforward and uses the projections of the joint mass matrix $\boldsymbol{M}(\boldsymbol{q})$ and the actual potential stiffness $\partial^{2} V_{\text {rep,total }}(\boldsymbol{q}) / \partial \boldsymbol{q}^{2}$ in repulsion direction for a mass-spring-damper relation. Compared to classical potential field based approaches, the force dependency of the dynamic constraints complicates the design because it leads to an implicit relation between load force (including the repulsive force) and distance to the boundaries. This can be easily interpreted: If the torso center point is close to a dynamic boundary and penetrates the potential field, a repulsive force is generated which increases the load on the joints and pulls this boundary even closer. That reduces the distance to the boundary and increases the repulsive 
force in turn. A local solution to that problem has to be found in order to apply a control law similar to (16). Based on the load force, the repulsive force from each kinematic constraint $i$ can be computed straightforwardly using the shortest distance $d_{i}$ and direction $\boldsymbol{n}_{i}$ to each boundary. The direction of the force is either pointing towards or away from the circle center, depending on the boundary. Due to the effect of the repulsive force on the dynamic constraints, they are resolved separately. Therefore, the worst case scenario with the maximum repulsive force $\boldsymbol{F}_{\text {rep,max }, i}$ from each dynamic boundary is considered. At each circular boundary $i$, the direction $\boldsymbol{n}_{i}$ is determined in the same way as for the kinematic constraints. At the straight boundaries, the force is directed perpendicular to the boundary into the workspace. The repulsive force increases the load on the joints. If it is considered as the counterforce to an additional load force $-\boldsymbol{F}_{\text {rep,max }, i}$, this results in change of the original load force $\boldsymbol{F}$ from (4) into a new load force

$$
\boldsymbol{F}_{\text {new }, i}=\boldsymbol{F}-\boldsymbol{F}_{\text {rep }, \max , i}
$$

which constructs new boundaries for $\tau_{2}$ and $\tau_{3}$. For the determination of the repulsive force from the dynamic boundaries, both sets of dynamic boundaries, those calculated with $\boldsymbol{F}$ and those calculated with $\boldsymbol{F}_{\text {new }, i}$ have to be considered. The relevant distance $d_{i}$ for the calculation of the force from each dynamic boundary $i$ is given by the minimum distance between the torso center point and the boundaries

$$
d_{i}=\min \left(d_{i}(\boldsymbol{F}), d_{i}\left(\boldsymbol{F}_{\text {new }, i}\right)\right) .
$$

However, the directions $\boldsymbol{n}_{i}(\boldsymbol{F})$ are taken from the calculation of the boundaries with $\boldsymbol{F}$. That choice solves the implicit problem mentioned above. The repulsive force from any kinematic or dynamic constraint is given by

$$
\boldsymbol{F}_{\text {rep }, i}=F_{\text {rep }}\left(d_{i}\right) \cdot \boldsymbol{n}_{i}
$$

where $F_{\text {rep }}\left(d_{i}\right)$ is related to the according potential $V_{\text {rep }}\left(d_{i}\right)$ by its gradient [10]. All single repulsive forces $\boldsymbol{F}_{\text {rep }, i}$ (kinematic, dynamic for $\tau_{2}$, dynamic for $\tau_{3}$ ) are added vectorially to determine the final direction of the overall repulsive force

$$
\boldsymbol{n}=\frac{\sum_{i} \boldsymbol{F}_{\text {rep }, i}}{\left\|\sum_{i} \boldsymbol{F}_{\text {rep }, i}\right\|_{2}} .
$$

The magnitude is defined by the overall minimum distance

$$
d_{\min }=\min _{i}\left(d_{i}\right)
$$

to the boundaries $i$ (kinematic and dynamic). Finally, the repulsive force is given by

$$
\boldsymbol{F}_{\text {rep }}=F_{\text {rep }}\left(d_{\text {min }}\right) \cdot \boldsymbol{n} .
$$

\section{EXPERIMENTS}

In this section, the controller (Sec. II-E) is applied and validated in two experiments.

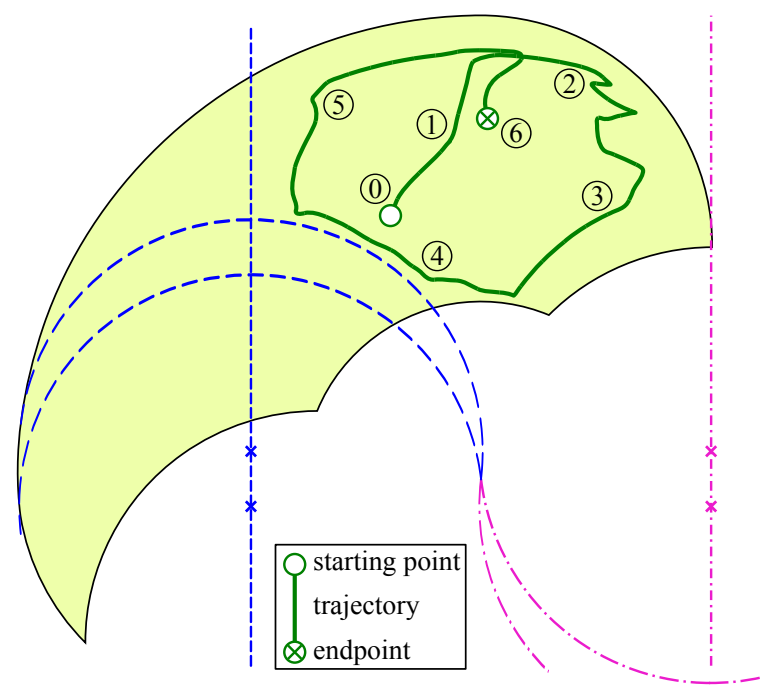

Fig. 5. Experiment \#1: In gravity compensation mode, the torso is manually moved in the $x-z$-plane without consideration of any constraints by the user himself. The torso center point stays within the admissible workspace due to active repulsion from the kinematic and dynamic boundaries. The instants (0) to (6) correspond to the ones in Fig. 6.

\section{A. Experiment \#1:}

This experiment is supposed to show how the controller forces the torso to stay in the admissible workspace. The user interacts with the robot in gravity compensation mode ${ }^{2}$ and moves the torso in the $x$-z-plane. The path of the torso center point is illustrated in Fig. 5. The damping ratio is set to $\xi=0.3$, the load force is specified by $\alpha=90^{\circ}$ and $F_{\text {load }}=470 \mathrm{~N}$. That load describes the weight of the whole upper body. The applied potentials have the form

$$
V_{\text {rep }}\left(d_{i}(\boldsymbol{q})\right)= \begin{cases}-\frac{f_{\text {max }}}{3 d_{\text {start }}^{2}}\left(d_{i}-d_{\text {start }}\right)^{3} & \forall d_{i} \leq d_{\text {start }} \\ 0 & \forall d_{i}>d_{\text {start }}\end{cases}
$$

with maximum force $f_{\max }=50 \mathrm{~N}$. The potential is nonzero for distances closer than $d_{\text {start }}=0.1 \mathrm{~m}$ to the boundary. This parameterization leads to a maximum stiffness in the potential of $1000 \mathrm{~N} / \mathrm{m}$ for $d_{i}=0 \mathrm{~m}$. The minimum and maximum torques are set to $\pm 140 \mathrm{Nm}$. An additional singularity avoidance is applied to avoid the outstretched torso configuration. The repulsive and damping forces along the path and the repulsion state of the controller are given in Fig. 6 as well as the torques measured at the second and third torso joint. During $41.5 \mathrm{~s} \leq t \leq 46.5 \mathrm{~s}$, one limit is slightly violated as $\tau_{3}<\tau_{3, \min }$. That is due to the assumed static force of $470 \mathrm{~N}$ while dynamic effects such as the externally applied forces by the user, inertial effects, and Coriolis/centrifugal forces are not considered in the control during the motion. However, that violation poses no problem because the limit torques of $\pm 140 \mathrm{Nm}$ have been set conservatively since the actuator limits in the torso joints of Justin are actually $\pm 230 \mathrm{Nm}$. That is one way to avoid the

\footnotetext{
${ }^{2}$ A gravity model is computed online and the gravitational effects are compensated.
} 

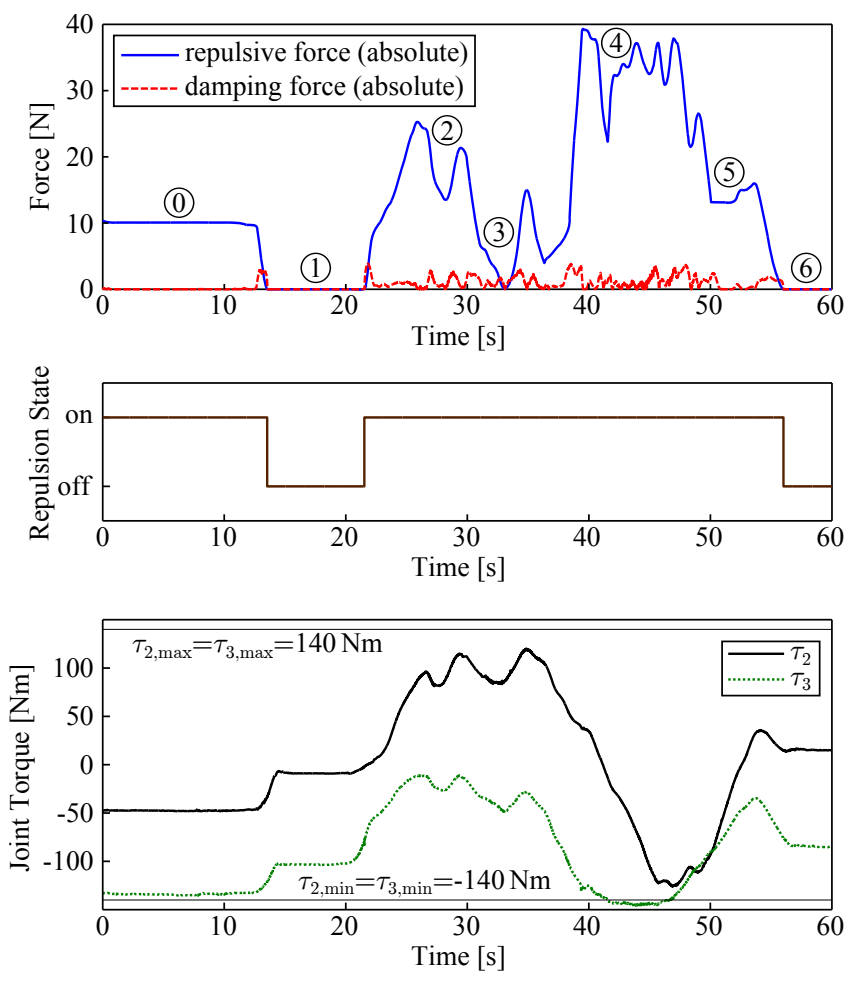

Fig. 6. Experiment \#1: The repulsive and damping forces corresponding to the path of the torso center point (Fig. 5) as well as the relevant joint torques are depicted. Except for two situations at about $t=20 \mathrm{~s}$ and $t=60 \mathrm{~s}$, the controller is permanently active due to the large potential field extent. The instants (0) to (6) correspond to the ones in Fig. 5.

numerically expensive computations of an online dynamic model of the robot as well as measurement of external forces.

\section{B. Experiment \#2:}

The same potential and load parameterization as in experiment \#1 is used here, but the potential field is larger now with a starting distance of $d_{\text {start }}=0.14 \mathrm{~m}$. Three damping ratios are compared. Fig. 7 and Fig. 8 depict the torso trajectory and the corresponding forces, respectively. The motion is realized by applying the same, fixed torque profile for each damping ratio additional to the controller from Sec. II-E. As expected, increasing the damping reduces the repulsion by tendency. The experiments show that a damping ratio $0.3 \leq \xi \leq 0.6$ leads to a proper motion behavior. A damping value in this range appears to be a good compromise between deliberate dissipation of kinetic energy and restriction of the dynamic capabilities of the torso.

\section{CONCLUSION}

The workspace and performance of a humanoid robot is significantly determined by the design of its torso. The typically high loads on the respective joints can be drastically reduced by kinematically coupling them. That, in turn, leads to configuration-based constraints on the system. Here, we analyzed these kinematic and dynamic constraints on the torso of the humanoid robot Rollin' Justin of the DLR. One application for that analysis, i. e. a potential field based

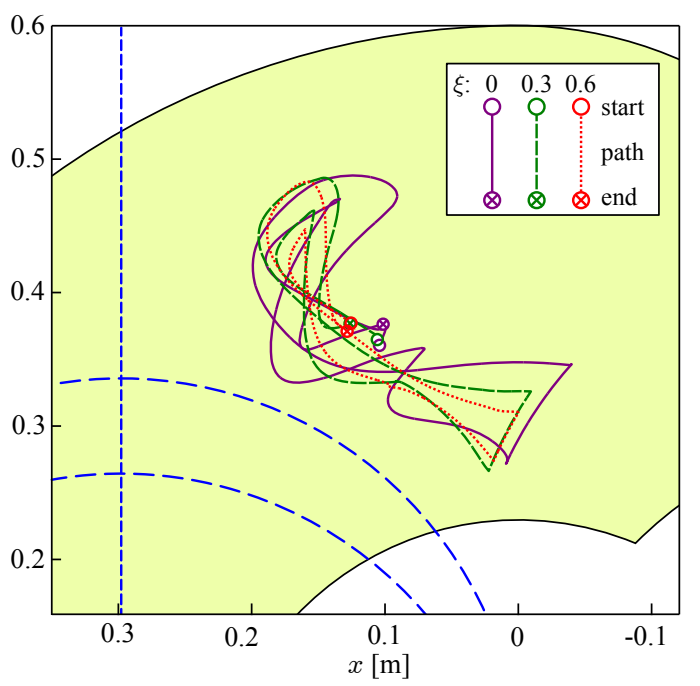

Fig. 7. Experiment \#2: Path of the torso center point for different damping ratios $\xi \in\{0,0.3,0.6\}$. A predefined torque profile triggers the motion. The corresponding forces are depicted in Fig. 8. The parameterization is: $F_{\text {load }}=470 \mathrm{~N}, \alpha=90^{\circ}, \tau_{2, \min }=\tau_{3, \min }=-140 \mathrm{Nm}, \tau_{2, \max }=\tau_{3, \max }=$ $140 \mathrm{Nm}$.
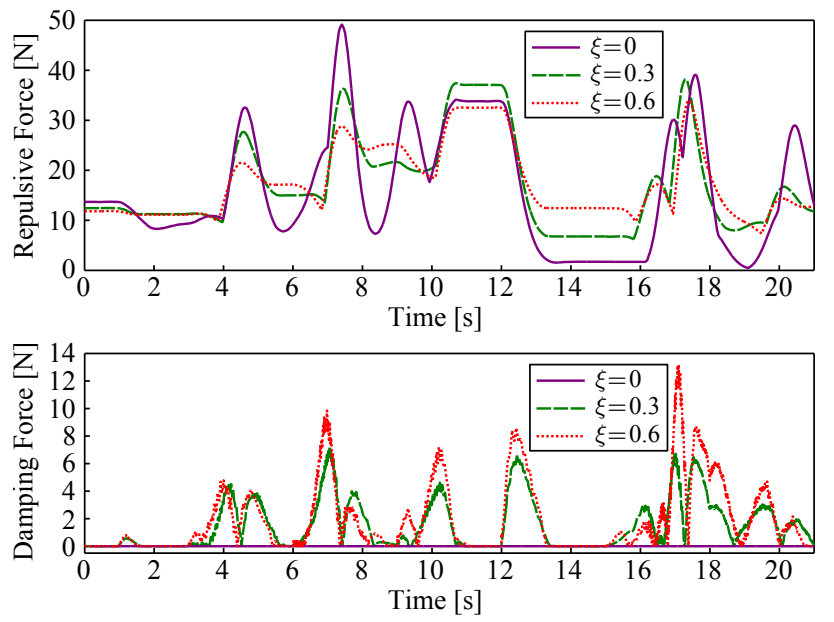

Fig. 8. Experiment \#2: The repulsive and damping forces for the three damping parameterizations $(\xi \in\{0,0.3,0.6\})$ in Fig. 7 are depicted.

controller to repel from these boundaries, has been designed, implemented, and evaluated on the robot.

\section{ACKNOWLEDGEMENTS}

This work was partially supported by the Helmholtz Alliance ROBEX and the ERC Starting Grant "Control based on Human Models" ERC-2013-StG 337654-CON_HUMO.

\section{REFERENCES}

[1] J. Or, "Humanoids Grow a Spine," IEEE Robotics \& Automation Magazine, vol. 20, no. 2, pp. 71-81, June 2013.

[2] H. Iwata and S. Sugano, "Design of Human Symbiotic Robot TWENDY-ONE," in Proc. of the 2009 IEEE International Conference on Robotics and Automation, May 2009, pp. 580-586.

[3] C. Borst, T. Wimböck, F. Schmidt, M. Fuchs, B. Brunner, F. Zacharias, P. R. Giordano, R. Konietschke, W. Sepp, S. Fuchs, C. Rink, A. AlbuSchäffer, and G. Hirzinger, "Rollin' Justin - Mobile Platform with Variable Base," in Proc. of the 2009 IEEE International Conference on Robotics and Automation, May 2009, pp. 1597-1598. 
[4] I. Mizuuchi, T. Yoshikai, Y. Nakanishi, Y. Sodeyama, T. Yamamoto, A. Miyadera, T. Niemelä, M. Hayashi, J. Urata, and M. Inaba, "Development of Muscle-Driven Flexible-Spine Humanoids," in Proc. of the 5th IEEE-RAS International Conference on Humanoid Robots, December 2005, pp. 339-344.

[5] F. Zacharias, C. Borst, M. Beetz, and G. Hirzinger, "Positioning Mobile Manipulators to Perform Constrained Linear Trajectories," in Proc. of the 2008 IEEE/RSJ International Conference on Intelligent Robots and Systems, September 2008, pp. 2578-2584.

[6] R. W. Ellenberg, R. Vallett, R. J. Gross, B. Nutt, and P. Y. Oh, "Development of the Skewed Rotation Plane (SRP) Waist Joint for Humanoid Robots," in Proc. of the 2013 IEEE International Conference on Technologies for Practical Robot Applications, April 2013, pp. 1-6.

[7] I. Mizuuchi, Y. Nakanishi, Y. Sodeyama, Y. Namiki, T. Nishino, N. Muramatsu, J. Urata, K. Hongo, T. Yoshikai, and M. Inaba, "An Advanced Musculoskeletal Humanoid Kojiro," in Proc. of the 7th IEEE-RAS International Conference on Humanoid Robots, December 2007, pp. 294-299.

[8] C. Ott, O. Eiberger, W. Friedl, B. Bäuml, U. Hillenbrand, C. Borst, A. Albu-Schäffer, B. Brunner, H. Hirschmüller, S. Kielhöfer, R. Konietschke, M. Suppa, T. Wimböck, F. Zacharias, and G. Hirzinger, "A Humanoid Two-Arm System for Dexterous Manipulation," in Proc. of the 6th IEEE/RAS International Conference on Humanoid Robots,
December 2006, pp. 276-283.

[9] T. Wimböck, Controllers for Compliant Two-Handed Dexterous Manipulation. Shaker Verlag, 2013.

[10] O. Khatib, "Real-Time Obstacle Avoidance for Manipulators and Mobile Robots," International Journal of Robotics Research, vol. 5, no. 1, pp. 90-98, Spring 1986.

[11] A. Dietrich, T. Wimböck, A. Albu-Schäffer, and G. Hirzinger, "Integration of Reactive, Torque-Based Self-Collision Avoidance Into a Task Hierarchy," IEEE Transactions on Robotics, vol. 28, no. 6, pp. 1278-1293, December 2012.

[12] C. Ott, Cartesian Impedance Control of Redundant and FlexibleJoint Robots, ser. Springer Tracts in Advanced Robotics. Springer Publishing Company, Berlin Heidelberg, 2008, vol. 49.

[13] A. Dietrich, T. Wimböck, and A. Albu-Schäffer, "Dynamic WholeBody Mobile Manipulation with a Torque Controlled Humanoid Robot via Impedance Control Laws," in Proc. of the 2011 IEEE/RSJ International Conference on Intelligent Robots and Systems, September 2011, pp. 3199-3206.

[14] A. Dietrich, T. Wimböck, A. Albu-Schäffer, and G. Hirzinger, "Reactive Whole-Body Control: Dynamic Mobile Manipulation Using a Large Number of Actuated Degrees of Freedom," IEEE Robotics \& Automation Magazine, vol. 19, no. 2, pp. 20-33, June 2012. 